\title{
Research in lower middle income countries - recommendations for a national mental health research agenda in South Africa
}

\author{
J Chipps', S Ramlall² \\ 1Department of Nursing and Public Health, University of KwaZulu-Natal, Durban, South Africa \\ 2Department of Psychiatry, University of KwaZulu-Natal, Durban, South Africa
}

\begin{abstract}
In the current mental health environment in South Africa, the development of a relevant mental health research agenda poses several challenges. This paper provides a brief overview of the current state of published research in mental health and, using a translation research framework, makes recommendations for five strategic directions to be considered in the development of a national mental health research agenda.
\end{abstract}

Keywords: LMIC; Research; Psychiatry; Mental health; South Africa

Received: $29 / 06 / 2012$

Accepted: 19/09/2012

doi: http://dx.doi.org/10.4314/ajpsy.v15i6.55

This article is based on a paper presented at the National Mental Health Summit convened by the Department of Health, Republic of South Africa from 12-13 April 2012.

\section{Introduction}

South Africa, similar to other lower middle income countries (LMICs), has a high burden of mental health problems ${ }^{1}$ and most mental health care users experience a substantial treatment gap. ${ }^{2-5}$ Services for people with mental health problems in the public sector have been affected by a shortage of skilled personnel, poor service provision with outdated psychiatric hospitals, difficulties in developing tertiary level specialist psychiatric services and underdeveloped community and psychosocial rehabilitation services. ${ }^{4}$ In addition, mental health services are not equitably distributed, with specialists localized to urban areas, usually around academic centers ${ }^{6}$, and about 56\% working in the private sector. ${ }^{7}$

Mental health research, including research in mental

Correspondence

Ms J Chipps

Department of Nursing and Public Health, University of KwaZulu-Nata

Private Bag 7, Congella, 4013, South Africa

email: chipps@ukzn.ac.za health, psychiatry or psychology, is needed to inform the development of locally-relevant, cost-effective mental health services and to ensure that these services are evidencebased. ${ }^{8}$ In this environment, the development of a mental health research agenda presents a number of challenges and opportunities. Nationally there has been a decline in published clinical research in South Africa due to two "decades of uncoordinated policy, a systemic withdrawal of provincial health departments from research support, underfunding of the agency component of the Medical Research Council, and the poor positioning of health research in the recent initiatives rolled out on behalf of the Department of Science and Technology by the National Research Foundation." "9 Researchers believe that there is a lack of mental health research resources in South Africa ${ }^{10}$ and though South Africa has a small, effective group of highly skilled and internationally recognized mental health researchers, lack of investment in the training of new health researchers ${ }^{11}$, the relative neglect of mental health and the heavy demand of clinical service and teaching on the small core group of academic clinicians is seen as an impediment to progress. ${ }^{12}$

The aim of this paper is to provide an overview of the current state of published research in mental health in South Africa and, using a translation research framework, to suggest five strategic directions to be considered in the development of a national mental health research agenda. 


\section{Background}

In the early 1990s the South African Medical Research Council, the National and Provincial Departments, as well as the Centre for Health Policy at the University of Witwatersrand made various attempts to coordinate a national mental research agenda. In 1994 a process of Essential National Health Research was established with mental health and substance abuse being prioritized in the top ten. ${ }^{13}$ Further attempts to identify gaps in mental health research through the European Commission project called 'Methods for intervention on mental health in subSaharan Africa', were followed by a workshop in 1999, which identified mental health research priorities. ${ }^{13}$ Since then, mental health has been recognized as a subcategory of communicable diseases, evident in the publication of the National Health Research Committee ${ }^{12}$, a statutory body established by the Minister of Health in terms of the National Health Act, 2003 (Act No. 61 of 2003), Section 69(1), the MRC National Research Strategy 2005 - 201014, and the National Conference on Priority Setting for Health Research.

\section{Current state of published research in mental health in South Africa}

\section{Publications in psychiatry and mental health}

In 1997, 542 references for mental health research in South Africa up to May 1999 were reported in a research database; more than half of these were not original research articles. ${ }^{15}$ Fourie, et al (2001) reviewed psychiatric research published by South African-based authors during the years 1966-1997 and found that there were few collaborative research groups, few authors wrote more than one paper and most papers were published in medical journals. ${ }^{11}$ This was similar to the WHO findings that $57 \%$ of 114 LMICs contributed less than five articles to the international mental health indexed literature for a 10-year period (1993-2003). ${ }^{16}$ a dissemination workshop found that up until 2001, a significant amount of research had already been done in South Africa, but the bulk was descriptive or analytic research with limited interventions evaluations. ${ }^{13}$ Similarly, in World Psychiatric Association psychiatry journals prior to 2008, a search in Medline and the Institute of Scientific Information (ISI) did not find any psychiatric journal indexed from the African region in at least one of the two databases ${ }^{17}$ but there was some evidence that articles were being published in high profile journals. ${ }^{18}$ Recently there has been some improvement in output ${ }^{19}$ with psychiatry scientific outputs according to publication counts from South Africa moving from position 120 (1990-1994) to 21 $(2004-2008){ }^{20}$

\section{Priorities for mental health research}

Various studies have addressed the question as to whether mental health research in South Africa addresses locally relevant priorities. In 2000, Flisher et al conducted a cross sectional survey of South African Research Conferences, comprising 627 conference abstracts, and concluded that the reported mental health research was less than half $(267,43 \%)$ and did not address priority issues (78, 29\% addressed identified priority areas) ${ }^{21}$ In addition, of the 73 mental health papers, 33 (45\%) addressed topics in health problem research, 7 (10\%) aetiology research, 22 (30\%) intervention research, and 11 (15\%) operational and health systems research. ${ }^{21}$ In 2007, Lund et al ${ }^{22}$ identified the following national priorities for mental health: economic evaluations evidence base for interactions between mental health and other health conditions, trials examining the cost-effectiveness of a range of psychosocial and psychopharmacological interventions and mental health consequences of wider social interventions and routine monitoring of mental health. This was similar to the global priorities identified by Thomlinson health policy and systems research, where and how to deliver existing cost-effective interventions in a low-resource context, epidemiological research, child and adolescent mental disorders and substance use. ${ }^{23}$ Sharan et al, identified priorities for LMIC countries based on a framework of the burden of disease, social justice, and availability of funds, namely: epidemiology (burden and risk factors), health systems and social science, depression/anxiety, substance use disorders and psychoses; children and adolescents; women, and people exposed to violence/trauma. ${ }^{16}$ In 201 1, Petersen concluded that more intervention research is needed in South Africa, focusing on organizational and human resource mix requirements, cost-effectiveness of a culturally appropriate, task shifting and stepped care approach. ${ }^{3}$

\section{Recommendations for a national health research agenda}

Translational research is defined as 'comprehensive applied research that strives to translate the available knowledge and make it useful...'24 and includes a range of research activities, from epidemiological perspectives, clinical research and translating effective interventions into research and practice to the pivotal role for evidence synthesis. ${ }^{25}$ Using a translational framework for mental health research and strategic directions from the NSW Ministry of Health, Australia ${ }^{26}$, five strategic directions are suggested for the development of a national health research agenda.

\section{Facilitate the generation of high quality research that is} relevant to policy and practice in the following areas

a. Analytical and descriptive mental health epidemiological studies

The first nationally representative epidemiological survey of common mental disorders in South Africa, or indeed in Africa, the South African Stress and Health (SASH) survey ${ }^{1}$ established the importance of ongoing regular collaborative (nationwide, multi-site) epidemiological surveys to establish the actual mental health disease burden, mental health determinants, the status and evidence for mental health service planning and current mental health priorities.

b. Clinical Mental Health Research

To address issues of aetiology and cost-effective interventions in local contexts, researchers must conduct clinical research designed to meet the needs of clinicians and others who are making decisions about patients' care.22,27 There is a lack of controlled intervention studies undertaken outside the context of tertiary centers in the developed world. ${ }^{28}$ The importance of supporting and funding locally relevant clinical studies in neuroscience, psychopharmacological and psychotherapeutic interventions in LMICs was aptly illustrated in the study by Burns, which dispelled the myth, that the course and outcome of schizophrenia was better in developing countries. ${ }^{5}$ This finding necessitates 
a reappraisal of treatment strategies for LMIC, emphasizing that local clinical practice must be supported by locally relevant evidence.

c. Systematic reviews of mental health evidence The development of systematic reviews applicable to LMIC and with internal and external validity is essential. While current involvement in evidence-based centers such as the South African Cochrane Centre and the two Joanna Briggs Institute Affiliate Centres at the University of KwaZulu-Natal and Witwatersrand is encouraging, only three systematic reviews based ${ }^{29-31}$ and two current registered systematic review protocols ${ }^{31,32}$ on a mental health related topic were found relevant to these Centres to date.

2. Facilitate the generation of high quality surveillance and mental health service data relevant to policy and practice Routine data collection from all tiers of the health system is essential to report on baseline mental health indicators and should be included in service delivery agreements. An integrated national surveillance system and appropriate monitoring and evaluation systems for mental health care should be functioning in all mental health services. In LMICs specifically, it is essential to establish a national minimum mental health data set, which does not overburden the already stretched health workers, is based on ICD10 and includes standard measures for outcomes and economic data. ${ }^{33}$

3. Foster mental health research funding and links to promote partnerships between funded research bodies, clinical academics, clinicians and mental health care users It is essential to increase resources (human and technical) for mental health research. South African research is underfunded relative to the burden of psychiatric disorders ${ }^{22}$ and has problems attracting talented researchers to neuroscience and mental health research. There should be political will from Government ${ }^{34}$ to support the funding of Mental Health Service and Research in line with the WHO recommendations for $\mathrm{LMIC}^{35}$, and the Academy of Science of South Africa's recommendations for revitalizing clinical research in South Africa. ${ }^{36}$ The National Department of Health should ensure that the National Health Research Committee ${ }^{12}$ incorporates specific priorities for research in mental health, and that mental health participation in the recent national health research scholarship program are ensured. ${ }^{37}$ Lastly, collaborative funding initiatives of research between local and international institutions, Departments of Health and Universities and public and private partnerships should be fostered and supported.

\section{Improve translation of research into practice}

The planning and provision of mental health services should be evidence-based, include the translation of evidence into practice and the evaluation of implementations. ${ }^{19,28,38}$ To ensure the translation of evidence to policy, there should be a focus on evidence-based policy development which is subject to scientific scrutiny. ${ }^{28}$ Currently there is a lack of integration of academic research and service and policy priorities. It is essential to involve policy makers in the planning and conduct of mental health research ${ }^{35}$ and to involve researchers and clinicians in the development and evaluation of policy.

In a limited resource setting like South Africa conducting empirical pilot studies for research translation and new interventions is difficult. A way to address this is using implementation research to monitor and evaluate innovative new interventions. An example of this is the action research framework for the implementation and evaluation for telepsychiatry services in KwaZulu-Natal. ${ }^{39}$

\section{Build mental health research capacity}

One of the challenges facing mental health is the dual role of clinician and researcher resulting in the need to invest in ongoing research training at undergraduate, postgraduate and managerial levels. Due to the resource constraints in LMICs, possible strategies that could be employed are: integrating formal systematic reviews as part of MMED or Masters Programs in collaboration with the South African evidence-based centers, involving mental health students in small scale action research projects in the clinical context as part of the implementation of evidence based practice ${ }^{40}$, providing mental health research and information literacy training to health managers and improving access to evidence based summaries, guidelines and mental health research publications. ${ }^{28}$ In addition, more Centers such as the Alan J. Flisher Centre for Public Health should be established. Recent outputs from the Centre include publishing the findings of the international collaborative Mental Health and Poverty project, which aimed to develop mental health research capacity in four $\mathrm{LMICs}^{41}$, and launching the first public mental health postgraduate training program in Africa in 2012. ${ }^{42}$

\section{Conclusion}

There are a number of challenges and opportunities for mental health research in LMIC. ${ }^{28}$ Though some progress has been made on the national research front, the challenges facing mental health research are compounded by the welldocumented current difficult state of psychiatry in the country. ${ }^{4}$ To ensure the implementation and development of research in a stressed system, special focus and attention should be given to establishing a translational research agenda for mental health in this setting.

\section{References}

1. Herman AA, Stein DJ, Seedat S, Heeringa SG, Moomal H, Williams DR. The South African Stress and Health (SASH) study: 12-month and lifetime prevalence of common mental disorders. South African Medical Journal 2009;99(5 Pt 2):339-344.

2. Kohn R, Saxena S, Levav I, Saraceno B. The treatment gap in mental health care. WHO Bulletin World Health Organisation 2004;82(11):858-866.

3. Petersen I, Lund C. Mental health service delivery in South Africa from 2000 to 2010: one step forward, one step back. South African Medical Journal 201 1;101(10):751-757.

4. Burns JK. Mental health services funding and development in KwaZulu-Natal: a tale of inequity and neglect. South African Medical Journal 2010;100(10):662-666.

5. Burns JK. The Mental Health Gap in South Africa - a human rights issue. The Equal Rights Review 201 1;6:99-113.

6. Ramlall S, Chipps J, Mars M. Impact of the South African Mental Health Care Act No. 17 of 2002 on regional and district hospitals designated for mental health care in KwaZulu-Natal. South African Medical Journal 2010;100(10):667-670.

7. Lund C, Flisher AJ. Staff/population ratios in South African public 
sector mental health services. South African Medical Journal 2002;92(2):161-164

8. Yasamy MT, Maulik PK, Tomlinson M, Lund C, Van Ommeren M, Saxena S. Responsible Governance for Mental Health Research in Low Resource Countries. PLoS Med 2011;8(11):e1001126.

9. Purposeful support for health research in South Africa. South African Journal of Science 2012;108(5/6), Art. \#1268, 1 page. http://dx.doi.org/10.4102/saj.s.v108i5/6.1268.

10. Razzouk D, Sharan P, Gallo C, Gureje O, Lamberte EE, de Jesus Mari J, et al. Scarcity and inequity of mental health research resources in lowand-middle income countries: a global survey. Health Policy 2010;94(3):211-220.

11. Fourie J, Flisher AJ, Emsley RA, Stein DJ. Psychiatric research in South Africa: a systematic review of Medline publications. Curationis 2001;24(2):9-11.

12. National Health Research Committee. 2011: National Health Research Summit Report: Strengthening research for health, innovation and development in South Africa. In: Department of Health, editor. Pretoria 2012

13. Thom R. Mental health service policy, implementation and research in South Africa - are we making progress? South African Journal of Psychiatry 2004;10(2):32-37.

14. South African MRC. Research Strategy 2005 - 2010. 2005.

15. Thom RGM. Monograph 69. Mental Health Services: A Review of Southern African Literature, 1967 to 1999.: Centre for Health Policy, University of the Witwatersrand,. 2000.

16. Sharan P, Gallo C, Gureje O, Lamberte E, Mari JJ, Mazzotti G, et al. Mental health research priorities in low- and middle-income countries of Africa, Asia, Latin America and the Caribbean. The British Journal of Psychiatry 2009;1 95(4):354-363.

17. de Jesus Mari J, Patel V, Kieling C, Anders M, Jakovljevi M, Lam LC, et al. The 5/95 Gap on the dissemination of mental health research: The World Psychiatric Association (WPA) task force report on project with editors of low and middle income (LAMI) countries. African Journal of Psychiatry 2009;12(1):33-39.

18. Ipser JC, Stein DJ. Little mental disorder research in general medical journals in low- and middle-income countries. South African Medical Journal 2007;97(2):110-111.

19. Stein DJ, Szabo CP. Psychiatry research in South Africa: reason for cautious celebration? African Journal of Psychiatry 201 1;14(2):86.

20. Kahn M. A bibliometric analysis of South Africa's scientific outputs some trends and implications. South African Journal of Science 2011;107(1/2): 207.

21. Flisher AJ, Parry CD, Stein DJ. To what extent does South African mental health and substance abuse research address priority issues? South African Medical Journal 2000;90(4):378-380.

22. Lund C, Stein DJ, Corrigall J, Bradshaw D, Schneider M, Flisher AJ. Mental health is integral to public health: a call to scale up evidencebased services and develop mental health research. South African Medical Journal 2008;98(6):444-446.

23. Tomlinson M, Rudan I, Saxena S, Swartz L, Tsai AC, Patel V. Setting priorities for global mental health research. Bulletin of the World Health Organization 2009;87(6):438-446.

24. Narayan KM, Gregg EW, Engelgau MM, Moore B, Thompson TJ, Williamson DF, et al. Translation research for chronic disease: the case of diabetes. Diabetes Care. 2000;23(12):1794-1798.

25. Ogilvie D, Craig P, Griffin S, Macintyre S, Wareham NJ. A translational framework for public health research. BMC Public Health 2009;9:116-116.

26. NSW Ministry of Health. NSW Mental Health Research Framework Sydney, Australia.2012.
27. March JS, Silva SG, Compton S, Shapiro M, Califf R, Krishnan R. The case for practical clinical trials in psychiatry. The American Journal of Psychiatry 2005; $162(5): 836-846$.

28. Stein DJ, Seedat S. From research methods to clinical practice in psychiatry: challenges and opportunities in the developing world. International Review of Psychiatry 2007;19(5):573-581.

29. Cleary M, Hunt G, Matheson S, Siegfried N, Walter G. Psychosocial interventions for people with both severe mental illness and substance misuse. Cochrane database of systematic reviews (Online) 2008(1):CD001088.

30. Kaner EF, Beyer F, Dickinson HO, Pienaar E, Campbell F, Schlesinger C et al. Effectiveness of brief alcohol interventions in primary care populations. Cochrane database of systematic reviews (Online) 2007(2):CD004148.

31. Chipps J, Brysiewicz P. A systematic review of the experiences of videoconference telepsychiatry for health professionals and adult mental health care users in public mental health services (Protocol). In: The South African Centre for Evidence Based Nursing and Midwifery: An Affiliate Centre of the Joanna Briggs Institute. 2012. Availalble from: http://www.joannabriggs.edu.aul

32. Lewis IS, Joska JA, Siegfried N. Antidepressants for depression in adults with HIV infection (Protocol). Cochrance Database Syst ReV 2010 [serial on the Internet]. 201017 September 2012; (5): Available from: http://www.mrc.ac.za/cochrane/publicatons.htm.

33. Chipps J, Raphael B, Coombs T. The Mental Health Outcomes and Assessment Tools Training Project: Creating the foundation for improved quality of care. New South Wales Public Health Bulletin 2002;13(12):237-238.

34. Saraceno B, van Ommeren M, Batniji R, Cohen A, Gureje O, Mahoney J, et al. Barriers to improvement of mental health services in lowincome and middle-income countries. Lancet 2007;370(9593):1164-1174.

35. WHO. Research and Development to Meet Health Needs in Developing Countries: Strengthening Global Financing and Coordination. In: Consultative Expert Working Group on Research and Development: Financing and Coordination., editor. Geneva: WHO; 2012

36. Mayosi BM, Dhai A, Folb P. Revitalising Clinical Research in South Africa: A Study on Clinical Research and Related Training. Academy of Science of South Africa [serial on the Internet]. 2009: Available from: http://www.assaf.co.za/wp-content/uploads/2009/09/ASSAfClinicalReport-2009.pdf

37. Bateman C. Govt steps up to stop corrosion of health research. South African Medical Journal 2012;102(6):345-346.

38. Stein DJ, Daniels W, Emsley R, Harvey B, Blackburn J, Carey P, et al. A brain-behaviour initiative for South Africa: the time is right. Metabolic Brain Disease 2006;2 1 (2-3):279-284.

39. Chipps J, Ramlall S, Mars M. Developing telepsychiatry services in a resource constrained environment - an action research study in KwaZulu-Natal. African Journal of Psychiatry 2012; 15(4), 255-263.

40. du Toit SH, Wilkinson AC, Adam K. Role of research in occupational therapy clinical practice: applying action learning and action research in pursuit of evidence-based practice. Australian Occupational Therapy Journal 2010;57(5):318-330.

41. Lund C. Mental health in Africa: findings from the mental health and poverty project. International Review of Psychiatry 2010;22(6):547-549

42. Lucas P. New master's programme to build mental health care capacity in Africa UCT/SU joint initiative aims to help improve economy, development through mental health care. 2012; Available from: http://www.uct.ac.za/usr/press/2012/mental_healthcare.pdf. 